\title{
Lifesaving cancer surgeries need to be managed appropriately during the COVID-19 pandemic
}

The views expressed in this editorial are those of the authors and do not necessarily reflect the position of the Canadian Medical Association or its subsidiaries.

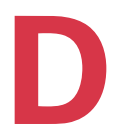

uring the coronavirus disease 2019 (COVID-19) pandemic, the number of cases and deaths have been overwhelming. Health care leadership and practitioners are increasingly faced with ethical and practical challenges.

Even as we all face the threat of this pandemic, an estimated 225800 Canadians will be diagnosed with cancer in 2020, and cancer is responsible for $30 \%$ of all deaths across the country. Modelling indicates that as many as 13000 people could be affected by a delay in access to cancer surgery over the first 3 months of the pandemic, and delays in cancer surgeries beyond 6 weeks can affect long-term outcomes for these patients. For others, a delay beyond 14 days may tip the scale. Thus, extreme caution is required in delaying lifesaving cancer surgeries. Postponed cancer surgery can also lead to conditions, such as bowel obstruction or spinal cord impingement, requiring emergent surgeries that otherwise could have been elective, often having a major impact on survival in these patients.

In Canada, many provinces have developed "cancer patient priority classification" systems to assist cancer programs in the management of patients. In addition to radiation and medical oncology, focused attention on the management of surgical oncology cases is required. To assist cancer surgeons and surgical administrative leaders in these difficult decisions and prioritization choices around the delivery of cancer surgeries among other competing pressures, the Canadian Partnership Against Cancer engaged the surgical oncology societies of Canada, cancer leaders and experts to provide their collective advice, guidance and methodologies for evidence- and risk-based decision making.

We have examined leading practices being used in some parts of Canada and internationally, and the accompanying guidance references a list of evidence-based and risk prioritization models. The collective advice can be summarized as follows:

- Cancer surgery must remain "essential" and should be among the last type of surgeries to be delayed.

- Transferring cancer surgery patients to less overwhelmed institutions should be the first-line strategy, requiring planning at a regional or jurisdictional level.

- If delays are necessary, they should be based on transparent jurisdiction-level clinical prioritization criteria.

- Plans should be in place to carry out staggered or delayed cancer surgeries within a reasonable time period, even if the pandemic is not over.

- Once the pandemic is over, there will be a surge in cases that we must anticipate, mitigate and plan for now.

We encourage that this guidance document be shared widely with colleagues engaged in the front-line decisions within institutions and health authorities.

Christian Finley, MD, MPH; Anubha Prashad, MBA;

Natasha Camuso, MSc; Corinne Daly, MSc;

Craig C. Earle, MD, MSc

Affiliations: From the Canadian Partnership Against Cancer (Finley, Prashad, Camuso, Daly, Earle).

Competing interests: None declared.

DOI: $10.1503 /$ cjs.005520 